http://dx.doi.org/10.18359/ravi.1862

\title{
Cómo empoderar a las instituciones de educación básica en la incorporación de las TIC ${ }^{1}$
}

\author{
Óscar Rafael Boude Figueredo ${ }^{3}$ \& Miguel Ángel Cárdenas Toro ${ }^{4}$ \\ Universidad de la Sabana, Colombia
}

Recibido, abril 27 de 2016

Concepto evaluación, mayo 20 de 2016

Aceptado, junio 30 de 2016
Referencia: Boude Figueredo, O.; Cárdenas Toro, M. (2016). "Cómo empoderar a las instituciones de educación básica en la incorporación de las TIC". Revista Academia y Virtualidad, 9, (2), 27-40

\section{Resumen}

Este documento presenta los resultados de una investigación que se desarrolló en la Universidad de La Sabana en el año 2015, cuyo objetivo fue establecer cómo contribuye una estrategia, diseñada dentro del proyecto "Educación a la nube", a empoderar a las instituciones de Educación Básica y Media de Bogotá a la incorporación de las TIC en los procesos de formación. Para ello, se planteó un estudio de corte mixto con un alcance descriptivo a través del diseño de estudio de casos múltiples tomando la institución educativa como unidad de análisis. La muestra estuvo conformada por 16 instituciones de diferentes localidades de Bogotá. Los principales resultados indicaron que la estrategia diseñada contribuyó a este ejercicio de empoderamiento por parte de las instituciones participantes en cuanto a la integración de las TIC a sus procesos de formación; sin embargo, esto no ocurrió en igual proporción para cada una de ellas debido, en gran medida, a la desigualdad en el nivel de competencias TIC de sus docentes y al dispar grado de apropiación de las TIC de parte de sus directivas.

Palabras clave: empoderamiento, estrategias de aprendizaje, Planes TIC, desarrollo profesional docente, escenarios de aprendizaje, competencias TIC.

\footnotetext{
${ }^{1}$ Artículo de carácter reflexivo que presenta los niveles de empoderamiento de las instituciones de educación básica, como resultado del trabajo de investigación del Proyecto Profesoral "Observatorio Informática Educativa", del Grupo de Investigación Proventus.

${ }^{3}$ Doctor Ciencias de la Educación; Profesor-Investigador, Maestría Informática Educativa. oscar.boude@ unisabana.edu.co

${ }^{4}$ Magíster en Informática Educativa y Profesor de la Universidad de La Sabana. miguelcarto@unisabana.edu. co
} 


\title{
Empowering Basic Education Institutions to incorporate ICTs
}

\begin{abstract}
This paper offers the results of a research developed at the Universidad de La Sabana in 2015, and the main goal is establishing the impact of the strategy in the project "Educación a la nube" in order to empower Bogota institutions of primary and secondary education to incorporate ICTs into their training processes. A mixed descriptive study was conducted using a multiple case examination and taking the educational institution as the analysis unit. The sample consisted by 16 institutions in different districts in Bogota. The main results indicate that strategy helped to empower the institutions involved in the integration of ICTs into their pedagogical processes; however, this did not occur in the same proportion for each institution largely due to uneven level of ICTs skills by their teachers and dissimilar ICTs acquisition degree by their school boards.
\end{abstract}

Keywords: empowerment, learning strategies, ICTs planning, professional development of teachers, learning scenarios, ICTs competences.

\section{Como acrescentar o poder das instituições de educação básica com a incorporação das TIC}

\section{Resumo}

Este documento apresenta os resultados de uma pesquisa desenvolvida na Universidade da Sabana no ano 2015, cujo objetivo foi: como contribui a estratégia traçada dentro do projeto "Educação à nuvem", ao incremento do poder das instituições de educação básica e média de Bogotá, com a incorporação das TIC nos processos de formação. Para tal fim, planteou-se um estudo de corte misturado com alcance descritivo através do desenho de estudo de casos múltiplos tomando a instituição educativa como unidade de análise. A amostra esteve conformada por 16 instituições de diferentes distritos de Bogotá. Os principais resultados indicaram que a estratégia traçada contribuiu a acrescentar o poder das instituições participantes na integração das TIC a seus processos de formação; no entanto, isso não aconteceu na mesma proporção para cada uma delas devido, em alto grau, à desigualdade no nível de competência em TIC dos seus docentes e ao grau de apropriação das TIC dos diretivos.

Palavras chave: incremento do poder, estratégias de aprendizagem, planes TIC, desenvolvimento profissional docente, cenários de aprendizagem, competências TIC. 


\section{Introducción}

Para nadie es un secreto que tanto en Colombia como en otros países latinoamericanos, cuando los diferentes gobiernos se han cuestionado sobre cómo mejorar la calidad de la educación, uno de los principales factores identificados se relaciona indudablemente con la calidad de los docentes con las que cuentan las instituciones de educación. Y tal y como indican Robalino Campos y Koner "[...] la mayoría de reformas educativas de los países latinoamericanos han optado por focalizar sus esfuerzos en la 'capacitación' de maestros, en ocasiones fuera de un contexto de políticas, estrategias y programas de formación permanente" (2006, p.7).

Lo anterior, parte del hecho de reconocer que los docentes constituyen uno de los factores más importantes del proceso de formación de los estudiantes, pues son ellos quienes están día a día con los estudiantes y proponen los procesos que éstos deben desarrollar para alcanzar sus competencias. Por tanto, tal y como afirma Murillo Torrecilla, no sería posible hablar de una mejora en la calidad de la educación, sin preocuparse por el desarrollo profesional docente. $\mathrm{Y}$ aunque es claro que el desempeño de un docente depende de una diversidad de factores, "en la actualidad hay consenso acerca de que la formación inicial y permanente de docentes es un componente de calidad de primer orden del sistema educativo" (Murillo Torrecilla; 2006: 11).

Tanto es así, que al revisar la literatura científica y especializada es posible observar una diversidad de ejemplos de planes de formación docente desarrollados por los diferentes países latinoamericanos, entre los que vale la pena mencionar: el "Proyecto Enlaces" desarrollado por el Ministerio de Educación de Chile; el "Proyecto Teleduc" desarrollado por la Universidad católica de Chile; el "Programa de Gestión y Dirección de Núcleos y Unidades Educativas" desarrollado en Bolivia por su ministerio de educación; los proyectos "A que te cojo Ratón" y "En
TIC Confío" desarrollados por el Ministerio de Educación de Colombia; el "Proyecto Maestr@s.com" del Ministerio de Educación de Ecuador, y "Educador del Siglo XXI" de Panamá.

No obstante, aunque son muchos los esfuerzos realizados por gobiernos e instituciones de educación encaminados a fortalecer el desarrollo profesional docente, la mayoría de estas experiencias no han logrado ser sostenibles en el tiempo (Cejudo, 2008; Correa, 2008; Silva et al., 2006; Lucena, Martín y Díaz, 2002; Galvis, 2008; Boude, 2012), debido a diversos factores, entre los que cabe resaltar que:

- por lo general, estos procesos están destinados a pequeños grupos dentro de una institución cuya exigencia para participar consiste en replicar el proceso de formación recibido con sus compañeros, lo cual regularmente no ocurre; y en el mejor de los casos, cuando se presenta, siempre es sobre aquellas cosas que aún se acuerda el docente que participó.

- estos procesos están más diseñados para el desarrollo de habilidades en el manejo de herramientas que a la transformación de las prácticas educativas.

- los docentes encuentran muchas dificultades para poder implementar lo aprendido debido a las políticas restrictivas de las instituciones o de las mismas secretarías de educación.

- propician los mismos problemas de la educación tradicional, reforzando el rol pasivo de los docentes y a fortalecer el rol jerárquico en el cual el estudiante -en este caso, el docente- depende de lo que su profesor le desee transmitir.

Lo anteriormente expuesto fue una muestra de las principales preocupaciones del proyecto "Educación a la Nube", pues -como se indicó antes el objetivo principal del proyecto-, era empoderar a las instituciones oficiales de Educación Básica y Media del Distrito Capital, a la incorporación de las TIC a sus procesos de formación, y como indican varios autores (Cejudo, 2008; Correa, 2008; Silva et al., 
2006; Lucena, Martin y Díaz, 2002; Galvis, 2008; Boude, 2012; Murillo Torrecilla, 2006; Robalino Campos y Koner 2006), esto no es posible si no se logra mejorar el desarrollo profesional de los docentes que trabajan en la institución.

No obstante, asimismo resulta importante recalcar que para lograr empoderar a una institución en la integración de tecnologías, es necesario realizar un proceso que involucre a las diferentes áreas de la institución, tal como han demostrado varios autores. Al respecto, en Colombia gracias al proyecto "Planes TIC" del Ministerio de Educación, existe una gran experiencia al respecto y se han logrado identificar algunos elementos que se deben tener en cuenta cuando se quieren transformar los procesos de una institución educativa (Londoño y Castillo Peña, 2012; Rivero, García y Ruíz, 2015; Aponte Correa, 2016).

Por consiguiente, para alcanzar los objetivos propuestos dentro del proyecto, se diseñó e implementó una estrategia enfocada a fortalecer los siguientes pilares dentro de cada institución educativa: el desarrollo profesional docente, la gestión académica, las competencias de los estudiantes y, por último, la interacción con la comunidad académica. En la siguiente sección se describirá el proceso seguido en la estrategia.

\section{Estrategia diseñada}

Tal y como se indicó con anterioridad, este proyecto plantea que para empoderar a una institución educativa en cuanto a la incorporación de las TIC a los procesos de formación, es necesario fortalecer el desarrollo profesional docente, los procesos de formación mediados por las TIC, la gestión académica, las competencias de los estudiantes $\mathrm{y}$, por último, la interacción con la comunidad académica.

Para lograrlo, se propuso una estrategia dividida en las siguientes fases: Planes TIC, Escenarios de aprendizaje, Producción de contenidos digitales y Visualización de experiencias, tal como se pueden apreciar en la figura 1.

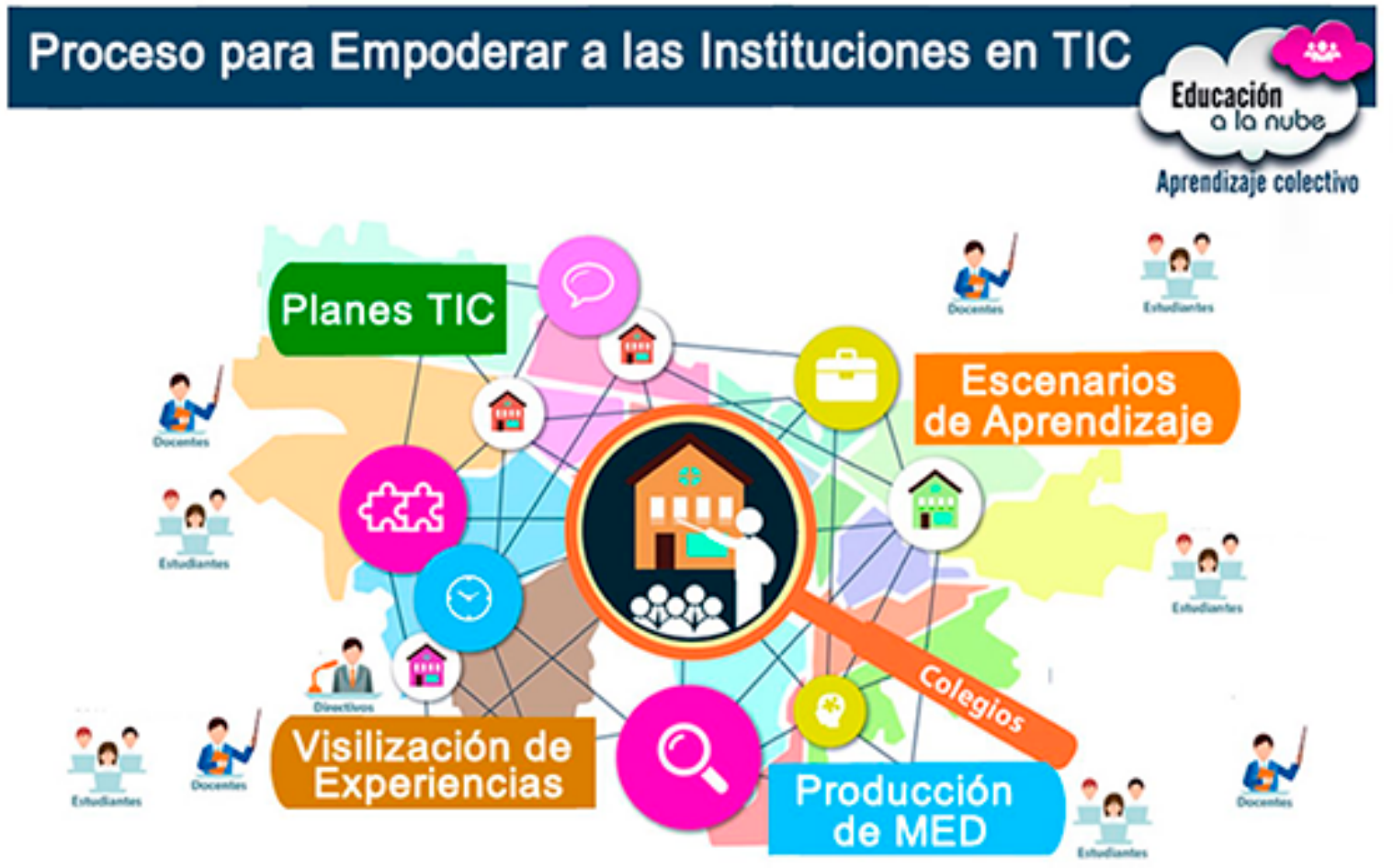

Figura 1. Estrategia diseñada para empoderar a las instituciones distritales de Educación Básica y Media de Bogotá. Fuente: CMED-CTA. 
Para su concepción y fundamentación, como referentes se tomaron los aportes realizados por diferentes investigadores alrededor del proceso de formación docente en lo que respecta a la integración de tecnologías de la información y de la comunicación, y su incorporación en todos los estamentos de la cotidianidad, el diseño de planes estratégicos en TIC, la construcción colectiva de conocimiento, el trabajo colaborativo, las exigencias de la sociedad del conocimiento (Castells, 1997; Marcelo, 2001; Lucena, Martín y Díaz, 2002; Hargreaves, 2003; Murillo Torrecilla, 2006; Robalino Campos y Koner 2006; Cejudo, 2008; Correa, 2008; Silva et al., 2006; Galvis, 2008; Salinas, Medina Rivilla, et al., 2011; Boude 2013,2012), y las propuestas realizadas por el Ministerio de Educación Nacional (2013), alrededor de las competencias TIC para el desarrollo profesional docente.

Asimismo, antes de continuar resulta importante precisar lo que para este proyecto se entendió por el concepto de empoderamiento. Según la definición del Diccionario de la Real Academia de la Lengua, la palabra empoderar es: "Hacer poderoso o fuerte a un individuo o grupo social desfavorecido" (RAE, 2013). Al aproximarnos a esta definición, se entiende que empoderar a una institución educativa para la integración de las TIC, sería lograr transformarla en una entidad que cuente con fortalezas alrededor de la integración de las TIC al proceso de formación. Otras aproximaciones como la de Silva y Loreto, quienes plantean que el empoderamiento desde el punto de vista de la psicología comunitaria "Ha sido señalado como una de las vías fundamentales para el desarrollo y la transformación de las comunidades" (2004, p.1), y desde esta mirada el empoderamiento de la institución educativa, resultaría fundamental si se quiere generar al interior de la entidad un proceso de transformación en la integración de las TIC al proceso de formación.

En otro sentido, tal como definen el empoderamiento Rubio y Escofet: “[...] un proceso de adquisición de poder de las personas que se encuentran en desigualdad respecto a otras [...] para tomar decisiones acerca de su vida, y ser partícipes y realizar cambios positivos en esta" (2013, p.1). Bajo esta postura, se interpreta que empoderar a una institución en la incorporación de TIC al proceso de formación, implicaría dotar de poder a la institución para tomar decisiones acerca de cómo desarrollar sus procesos, hacerlas partícipe de éstos y realizar los ajustes necesarios, de tal forma que se puedan generar cambios positivos.

Por otra parte, según Torres (2009), el empoderamiento se entiende como "[...] el proceso de concienciación que da cuenta al estudiante de sus capacidades desde lo cual potencia su acción para transformarse y transformar su contexto" (p.1), y esto implica, además, según el autor, "[...] el reconocimiento del poder del estudiante como elemento transformador de sí mismo y de su contexto" (p.1). Por consiguiente, empoderar a una institución educativa en la incorporación de las TIC a los procesos de formación, implicaría lograr que la institución tome conciencia de las capacidades que tiene para integrar estas tecnologías a sus procesos de formación y a partir de esto tomar las decisiones para transformarse y transformar su contexto.

Ahora bien, teniendo claridad sobre las implicaciones que tiene el empoderamiento de una institución educativa con la integración de las TIC, se formuló una estrategia dividida en cuatro fases denominadas: Planes TIC, Escenarios de aprendizaje, Producción de contenidos digitales y Visibilización de experiencias. Resulta importante asimismo mencionar que las dos primeras fases se desarrollaron de forma paralela en las instituciones de educación, mientras que la tercera se desarrolló a mediados del proyecto, y la última fase de principio a fin del proyecto. A continuación se describe el proceso en cada una de ellas. 


\section{Planes TIC}

Para el desarrollo de esta fase se solicitó a los rectores de cada institución la conformación de un equipo de trabajo de seis personas, así: dos directivos, dos docentes, un estudiante y el rector o su representante. Para su concepción se realizó un proceso de formación y asesoría in situ con la asesoría de un experto en el diseño de este tipo de planes estratégicos que buscó en todo momento que el PlanTIC formulado respondiera a las necesidades educativas del contexto y que tomara como punto de partida los planteamientos estipulados por la institución en su PEI, así como la visión y misión de la misma. Asimismo, resulta importante mencionar que una de las mayores dificultades que presentó este proceso es el desconocimiento por parte de todos los participantes del proceso que se debe seguir para generar un plan estratégico; por ello fue necesario realizar un plan estratégico base que sirviera de guía a los equipos de trabajo y sobre éste se pudiera generar el PlanTIC de la institución.

\section{Escenarios de aprendizaje}

Debido a que una de las dificultades más frecuentes, cuando se quiere implementar un plan estratégico relacionado con la incorporación de TIC (PlanTIC) dentro de una institución educativa, es que los docentes no están capacitados para desarrollar las metas que se han trazado en éste, por tanto, la segunda fase de esta estrategia denominada escenarios de aprendizaje estuvo encaminada a desarrollar tanto en los docentes como estudiantes de las instituciones participantes las competencias necesarias para que se pudieran desempeñar de forma adecuada en el siglo XXI. Para esto se diseñaron diferentes cursos orientados a fortalecer, por un lado, las competencias TIC planteadas por el MEN en $2013 \mathrm{y}$, por el otro, se diseñaron 20 cursos virtuales orientados a fortalecer el desarrollo de las competencias para el siglo XXI propuestas por la Unesco.

Particularmente, a los docentes se les capacitó en cuatro grandes áreas; la primera de ellas sobre la producción de contenidos educativos de calidad; la segunda, diseño de ambientes de aprendizaje mediados por TIC; la tercera, integración de LMS para el apoyo al proceso presencial; y la cuarta, generación de libros digitales. Vale asimismo mencionar que en estos cursos, los docentes estuvieron acompañados por cuatro de sus estudiantes; sin embargo, esto no fue posible en todas las instituciones pues muchos rectores no autorizaron su presencia para estar en la institución en un horario extra jornada.

\section{Producción de contenidos digitales}

Esta fase tenía como objetivo proveer a los docentes de todas las instituciones participantes en el proyecto de los recursos adecuados para transformar sus procesos de formación. Para esto, con ayuda de varios expertos se realizó un proceso de curación de 1500 contenidos educativos abiertos en las áreas de matemáticas, lenguaje, ciencias naturales y ciencias sociales, que se alojaron en un repositorio disponible para toda la comunidad académica a través de los servidores de la Secretaría de Educación de Bogotá. Asimismo, es importante mencionar que dentro de este repositorio se agregaron además recursos educativos creados por los docentes dentro del curso de producción de contenidos educativos de la segunda fase. Ahora bien, la mayor dificultad de esta fase, se relacionó con la selección de los contenidos, pues fue necesario revisar 5500 contenidos para poder seleccionar los 1500 adecuados para las necesidades del país.

\section{Visibilización de experiencias}

La cuarta fase de todo el proyecto propuso la generación de comunidades académicas a través de una serie de encuentros presenciales en 10 de las localidades de Bogotá donde se fomentó la socialización de las experiencias más significativas de cada una. Asimismo, se propuso la creación de un portal educativo para cada institución de educación, de tal forma 
que luego de la socialización de experiencias y la identificación de experiencias por parte de los docentes, este portal sirviera para que los maestros socializaran sus experiencias ante la comunidad académica.

Sin embargo, en esta fase se presentaron las mayores dificultades en el proyecto, pues no fue posible contar con asistencia de muchos docentes, ya que los rectores no les dieron permiso para asistir y los pocos presentes debían ir con sus alumnos. En el mismo sentido, se tuvieron problemas al montar los portales educativos en línea, ya que aunque se crearon, no fue posible subirlos desde las instituciones de educación, por problemas en la red de la Secretaría de Educación.

\section{Método}

El estudio se basó epistemológicamente sobre el paradigma interpretativo de la investigación; éste buscó comprender, describir e interpretar la realidad (Canales, 2006). Para el caso particular del proyecto, la realidad que se pretendió interpretar fueron las prácticas, las interacciones y las competencias que se generaron dentro de las instituciones distritales de Educación Básica y Media de Bogotá, al formar parte de la estrategia diseñada en el proyecto "Educación a la nube". Se utilizó un diseño mixto para lograr el objetivo. La contribución de la estrategia, diseñada dentro del proyecto citado, al empoderamiento en la incorporación de las TIC a los procesos de formación en las instituciones de Educación Básica y Media de Bogotá, y se desarrolló un estudio de casos múltiples con una muestra acordada con la Secretaría de Educación Distrital. De igual manera, se utilizaron instrumentos cuantitativos, no con el fin de cualificar los datos cuantitativos, por el contrario, se utilizaron los hallazgos cuantitativos y cualitativos para lograr, como indican Tood, Nerlich y McKeown (2004) en (Hernández, Fernández \& Baptista, 2010), una perspectiva más precisa del fenómeno.

El estudio tiene un alcance descriptivo- exploratorio; aquí se utilizaron instrumentos de línea base pre-post, pruebas iniciales y finales. Aunque en el país existen procesos diseñados para formación de docentes en integración de las TIC, desarrollo de portales educativos y generación de contenidos educativos digitales, éstas han sido iniciativas individuales y no se han diseñado proyectos encaminados a empoderar a los docentes de Educación Básica y Media para la integración de las TIC y a nivel cualitativo se realizará un estudio descriptivo a fin de comprender las opiniones de los docentes en cuanto a la estrategia diseñada.

\section{Participantes}

La población que participó en la investigación estuvo conformada por 160 instituciones de Educación Básica y Media de Bogotá, las cuales pertenecían a 13 diferentes localidades del Distrito, entidades que hicieron parte del convenio entre la Secretaría de Educación y la Universidad de La Sabana. Inicialmente se decidió tomar una muestra de 63 instituciones, pero en el momento de la recolección de datos hubo inconvenientes, por ejemplo la negativa por parte de los rectores y/o docentes para completar pruebas iniciales (pre-) o a las entrevistas, generando así una disminución de 50\%, 32 instituciones. Sin embargo, al revisar los datos recolectados dentro de los instrumentos, fue posible evidenciar que varios de los docentes y directivos participantes habían indicado que sus datos no se incluyeran en la investigación, generando así que no se alcanzara la muestra mínima de cuatro registros de cada instrumento. Finalmente, de las 63 instituciones iniciales, la muestra de este estudio estuvo conformada por 16 instituciones educativas distritales que entregaron la totalidad de instrumentos aplicados durante la investigación y que además dieron su consentimiento para que los datos pudieran ser tenidos en cuenta dentro de la investigación.

\section{Instrumentos de medida}


La recolección de los datos se llevó a cabo a través de instrumentos disponibles en la web, instrumentos aplicados físicamente en cada institución de Educación Básica y Media participante en el estudio, con el fin de reunir las evidencias que les permitieron a los investigadores, comprender los comportamientos, las maneras de hacer, las percepciones, las concepciones y las reflexiones que llevaron a los diferentes actores de cada institución participante a desarrollar la capacidad de incorporar las TIC a los procesos de formación de la entidad educativa. Estas evidencias fueron recolectadas a través del trabajo de campo, utilizando principalmente técnicas y análisis de tipo cualitativo, con el apoyo de algunas técnicas cuantitativas.

Para la identificación del nivel de competencias de los docentes, se diseñó y validó una prueba pre-post a través de la aplicación del mismo con 98 docentes de la Secretaría de Educación Distrital, que estaban cursando las maestrías ofrecidas por el Centro de Tecnologías para la Academia de la Universidad de La Sabana; luego, se aplicó el Alfa de Cronbach a la prueba y el coeficiente Alfa dio 0.95. El instrumento se aplicó al inicio del proyecto y tres semanas después de finalizar el proceso en cada institución.

Otro de los datos recolectados fue el seguimiento de todo el proceso; para esto se diseñó un instrumento que permitió recoger información de los participantes alrededor de cómo se había desarrollado la estrategia en cada institución, con el fin de identificar el efecto que tuvo en cada institución la forma particular como cada formador implementó in situ la estrategia diseñada. Para recolectar estos datos se realizó una encuesta estructurada, la cual fue digitalizada a través de la plataforma de Google Drive. Asimismo, para contrastar los datos recolectados en el instrumento de seguimiento con las percepciones de los participantes, se realizaron una serie de entrevistas estructuradas in situ con docentes de diferentes colegios participantes
Así como el plan estratégico en integración de TIC-que debió formular cada institución como ruta de navegación para poder integrar las TIC a los procesos de formación-, los productos generados por los docentes y las observaciones no participantes, que permitieron tener una visión general de lo que ocurrió en cada entidad y poder dar respuesta a interrogantes que surgieran al respecto durante el proceso de análisis de los resultados y que no estuvieran en los instrumentos recolectados.

\section{Métodos de análisis de datos}

Los datos cuantitativos se analizaron a través de Microsoft Excel y los datos cualitativos mediante ATLAS.ti versión 7. Para el análisis se codificaron los datos recolectados en los diferentes instrumentos, entrevistas, productos generados por los docentes participantes y el objetivo de la investigación, y se definieron las siguientes categorías a priori, con el fin de observar los diferentes actores que interactuaron en el proyecto por parte de cada institución educativa: Planes TIC, Escenarios de aprendizaje, Producción de contenidos y Visibilización de experiencias.

En la categoría PlanTIC, se tomaron datos provenientes de los Planes TIC y se segmentaron en las siguientes categorías emergentes: percepciones sobre el Plan TIC, Plan TIC desarrollado, visión y direccionamiento estratégico, construcción del conocimiento, y alcance, infraestructura y recursos.

En la categoría Escenarios de Aprendizaje, se identificó el efecto que tuvieron los escenarios de aprendizaje del proyecto dentro del empoderamiento de las instituciones de educación. Los datos se segmentaron en las siguientes categorías emergentes: elementos que contribuyen al empoderamiento, dificultades en el proceso de formación.

Asimismo, en la categoría Producción de contenidos, se analizaron los diferentes productos desarrollados por los docentes y se encontraron evidencias que permitieron determinar su utilidad para apoyar los 
procesos de formación mediados por TIC en la institución, así como su relación con lo propuesto dentro del Plan TIC. A partir de esta categoría surgieron las siguientes categorías emergentes: Desarrollo de cursos virtuales con Moodle, desarrollo del libro e-Pub, estándar de producción de un material educativo digital, y el diseño y producción de una web educativa. Para finalizar, en la categoría Visibilización de experiencias, se identificó el efecto que tuvieron los diferentes escenarios desarrollados dentro de esta fase en la conformación de redes o espacios de intercambio con la comunidad académica que ayuden a alcanzar las metas propuestas en el Plan TIC.

\section{Aspectos éticos}

Durante la investigación se tuvieron en cuenta todas las normativas existentes en la Universidad de La Sabana a fin de garantizar el anonimato de los participantes. En los instrumentos se remplazaron todos los nombres por códigos. De esta forma, aunque se pueda identificar la institución educativa, no se podrán revelar los docentes participantes.

Así mismo, para el estudio sólo se tuvieron en cuenta los datos de los docentes que dieron su consentimiento informado en todos los instrumentos utilizados. Si en algunos de ellos el docente expresaba no dar su aval inmediatamente, todos los datos de ese profesor o directivo se retiraban del estudio, sin leer algo de lo reportado por éste.

\section{Resultados}

Para la presentación de los resultados dentro del texto se estructuraron dos apartados diferentes. En el primero se describen las características que presentaron las Instituciones Educativas de Básica y Media que lograron empoderarse y en el segundo, las características de las entidades educativas que alcanzaron un empoderamiento parcial.

Antes de continuar, es importante mencionar la forma como se analizaron los casos y las variables utilizadas para determinar que una institución quedó empoderada para integrar las TIC a los procesos de formación.

Cada uno de los 16 casos realizados se analizaron de forma individual por los investigadores en función de las categorías de análisis: Plan TIC, Escenarios de aprendizaje, Producción de contenidos y Visibilización de experiencias. Esto con el fin de identificar evidencias que permitieran indicar el grado de empoderamiento de la institución con base en los siguientes criterios:

- La institución cuenta con un plan estratégico en integración de las TIC que responda a las necesidades de su contexto y a lo establecido en su PEI.

- La institución cuenta con contenidos educativos de calidad desarrollados por los docentes.

- $\quad$ La institución cuenta con un portal educativo que le permite mostrar los resultados de sus proyectos educativos con la comunidad académica.

- La institución cuenta con una comunidad que está convencida y comprometida con la integración de las TIC a los procesos de formación.

- La institución cuenta con autoridades académicas que apoyan la incorporación de las TIC a los procesos de formación.

- Docentes con niveles de competencias que permitan descender lo establecido en el PlanTIC.

- Docentes que durante el proceso de formación hayan realizado transformaciones de sus prácticas pedagógicas mediante la incorporación de las TIC.

A partir de los datos recolectados en los diferentes instrumentos fue posible establecer la cantidad de instituciones que quedaron empoderadas y aquellas que no alcanzaron a estarlo, tal como se indica en la tabla 1. 
Tabla 1. Empoderamiento de las instituciones educativas

\begin{tabular}{l|c|c}
\hline Nivel de empoderamiento & Cantidad & Representación \\
\hline Instituciones empoderadas & 11 & $69 \%$ \\
\hline Instituciones parcialmente empoderadas & 5 & $31 \%$ \\
\hline Instituciones no empoderadas & 0 & $0 \%$ \\
\hline
\end{tabular}

A continuación se hace una descripción de las características identificadas dentro del análisis a las instituciones empoderadas y a las parcialmente empoderadas.

\section{Características de las instituciones empoderadas}

Los resultados del estudio revelan que bajo este apartado se agruparon el $69 \%$ de las instituciones participantes en la investigación, 11 entidades educativas de Básica y Media. Éstas se caracterizaron por tomar conciencia de la incorporación de las TIC a los procesos de formación y esto quedó plasmado en el documento de Plan TIC que elaboraron, a través, de la visión, los objetivos estratégicos, la relación con el modelo pedagógico y el alcance de las TIC. Así como a través de la planeación de los recursos necesarios para la adecuada implementación del Plan TIC.

En el mismo sentido, las instituciones se caracterizaron por contar con un grupo de docentes con un nivel de competencias TIC para el desarrollo profesional docente, de acuerdo a los alcances plasmados en el Plan TIC. Asimismo, aquellas que quedaron con recursos educativos digitales, tales como cursos virtuales para apoyo al proceso presencial $\mathrm{y}$ contenidos educativos digitales que se puedan utilizar por parte de los profesores en su práctica docente. Estas instituciones se caracterizaron por el compromiso que existió por parte de directivos y docentes participantes durante el proceso de formación, así como después de éste para dar continuidad al plan elaborado en la institución. Esto implicó, en la mayoría de los casos, cambios de percepción en los paradigmas de los docentes sobre cómo se deben integrar las TIC a los procesos de formación.

Para finalizar también hubo instituciones empoderadas que ya tenían potencial para serlo antes de la implementación del proyecto. Instituciones de educación del distrito, que antes de desarrollar el proyecto "Educación a la nube", ya tenían el potencial necesario como entidad empoderada, por el nivel de desarrollo de competencias en TIC de sus docentes o por la participación previa en proyectos similares organizados por la Secretaría de Educación Distrital, , pero que no contaban con una ruta clara sobre la cual encauzar ese potencial. Entonces, luego de analizar los datos provenientes de estas instituciones, se decidió generar un nivel diferente de empoderamiento, es decir, un nivel intermedio, pues no se podía afirmar con total seguridad, que los resultados obtenidos por estas instituciones fueran producto del proyecto "Educación a la nube".

No obstante, en la investigación se logró establecer que el proyecto les ayudó a estas últimas instituciones a generar el Plan TIC, el cual sirvió en todos los casos como estrategia adecuada para canalizar el potencial con el que contaban y, a partir de este, definir los objetivos estratégicos trazados a nivel de integración de las TIC y los procesos que necesitarían desarrollar para alcanzar lo proyectado. 


\section{Características de las instituciones parcialmente empoderadas}

En este apartado se agruparon el $31 \%$ de las instituciones participantes en la investigación, cinco de Básica y Media. Éstas se tipificaron por no alcanzar una coherencia entre todas las características para empoderar a la entidad educativa en cuanto a la incorporación de las TIC a sus procesos de formación. Por un lado, fueron aquellas instituciones en las que no se identifica una coherencia en lo que respecta a los diferentes apartados del Plan TIC, que -aunque formulan la visión y objetivos estratégicos pensando en la incorporación de TIC dentro de toda la institución cuando se analiza su alcance- se limita escasamente al área de tecnología, sin que en las propuestas se identifique cómo afectará el currículo de las diferentes áreas, y esto queda evidenciado como un enfoque exclusivo para el currículo de tecnología.

Por otro lado, se agruparon aquellas instituciones en las que no se tuvo en cuenta su modelo pedagógico para planear cómo se debía realizar la incorporación de las TIC a los procesos de formación y, por ende, no se identificó cómo se debió realizar este proceso en el nivel curricular. En el mismo sentido, se agruparon otras entidades en las que no se desarrolló a totalidad el Plan TIC, quedando la última o últimas dos cartillas, de 5 , sin formular en su totalidad. Sin embargo, éstas cuentan con docentes con un nivel de competencias en TIC que les permitiría completar el Plan TIC. Es posible por falta de tiempo que haya ocurrido esto. En cuarto lugar, aquellas instituciones que, aunque diseñaron un muy buen Plan TIC, sus docentes no alcanzaron los niveles adecuados para que los alcances y objetivos estratégicos se pudieran alcanzar con facilidad dentro de la institución.

Asimismo, dentro de este apartado se agruparon las instituciones en las cuales se pudo observar a docentes y directivos comprometidos con el proceso, pero no contaron con un rector que lograra cambiar sus paradigmas en torno a lo que implicaría integrar las TIC al proceso de formación. En este tipo de entidades es posible que los docentes, no contaran con el tiempo suficiente para desarrollar todas las actividades propuestas en el Plan TIC. Además, en éstas generalmente los rectores no participaron activamente en el diseño y construcción del Plan TIC y lo delegaron a un directivo.

\section{Conclusiones}

Al revisar los diferentes informes alrededor de la incorporación de las TIC a los procesos de formación por parte de organizaciones tales como la UNESCO (2006), los aportes realizados por Mejía (2014) y otras propuestas como las de Prieto (2013), es posible observar cómo la capacitación y el fortalecimiento de las competencias en TIC, apoyadas de diagnósticos y reflexiones de manera crítica sobre el rol docente, permitirán que los docentes fomenten en los estudiantes el uso de las TIC con actitudes y competencias que transformen el ámbito educativo.

A nivel nacional, el Plan Decenal de Educación 2006-2016 expone que no resulta suficiente capacitar a los docentes, sino que de manera imprescindible, se solicita a las instituciones educativas el diseño y la elaboración de currículos teniendo en cuenta las TIC como eje transversal junto con la promoción de la calidad de los procesos educativos y la permanencia de los estudiantes, fomentando de manera particular el aprendizaje autónomo colaborativo y el pensamiento crítico a través del uso de estas herramientas.

La primera conclusión se relaciona con la respuesta al objetivo de la investigación, pues el análisis realizado a los datos recolectados en cada uno de los casos de estudio, sugirieron que la estrategia utilizada dentro del proyecto "Educación a la nube", contribuyó a empoderar a la mayoría de las instituciones de educación participantes. Estos resultados son muy alentadores en varios aspectos ya que están alineados con otros estudios (Bennett y Daniel, 1999; y Swain, 2006) que abordan 
la formación profesional docente de base y posibilitan que las instituciones educativas sean empoderadas en la incorporación de las TIC a sus procesos de formación.

Asimismo, uno de los principales hallazgos realizados en este proyecto, sugiere que exista una relación directamente proporcional entre el nivel de empoderamiento al que puede llegar una institución y el compromiso que tiene la institución educativa durante el proceso. Tal como lo señalan Karsenti y Gauthier (2006), “[...] lo importante es la utilización juiciosa y pedagógica de las TIC en la enseñanza según las finalidades de la escuela" (p. 4). Ya que, no sólo basta con que la mayoría de los docentes o directivos participantes estén comprometidos con la transformación de los procesos de formación a través de la mediación de la tecnología, si de esto no está convencido el rector, ya que el resultado al final del proceso, es una institución educativa que está parcialmente empoderada, pues aunque cuenta con un Plan TIC que sirve de ruta de navegación, y con directivos y docentes con las competencias necesarias para desarrollarlo, tienen un rector que no ve este plan como algo prioritario para la institución educativa. De igual modo, si el rector y los directivos de la institución están comprometidos con transformar los procesos de incorporación TIC, pero los docentes participantes no lo están, el resultado de esto será una institución parcialmente empoderada, pues la ruta de navegación con la que cuentan sólo responde a las visiones de ellos, ya que, aunque participen los docentes durante el proceso, no se obtendrán los resultados que espera el directivo.

Ahora bien, cuando se logró compromiso por parte de todos los actores de la comunidad académica participantes en el proyecto, fue posible observar que las instituciones de educación se empoderaran al final del proceso, aunque durante éste no se logre que todos trabajen de forma sinérgica. Esto se debe, entre otras razones, a que cada uno está comprometido con su proceso y cuando requiera de los demás, la responsabilidad propia durante el proceso de ejecución de los Plan TIC. Al terminar el proyecto, la mayoría de las instituciones de educación (11 de 16) participantes quedaron empoderadas en cuanto a la incorporación de las TIC a los procesos de formación, así: construyeron un Plan TIC, fortalecieron las competencias en TIC de sus docentes, desarrollaron recursos educativos y favorecieron el intercambio de experiencias entre las mismas entidades educativas.

En consecuencia, gracias al proyecto "Educación a la Nube", las instituciones educativas distritales quedaron con una ruta de navegación, que les permitió incorporar las TIC a sus procesos educativos, de tal manera que, a partir de la finalización del proyecto, cada entidad debió focalizar sus esfuerzos para sostener a través del tiempo y mejorar el Plan TIC, identificando todas las oportunidades año a año de incorporar estas herramientas en sus procesos de formación.

\section{Referencias}

Boude Figueredo, O. R. (2012). Desarrollo de competencias genéricas y específicas en educación superior a través de una estrategia didáctica medida por TIC. Tesis doctoral. Universidad de Educación a Distancia. Recuperado de http://espacio.uned.es/fez/ eserv.php?pid=tesisuned:EducacionOrboude $\& d s I D=$ Documento.pdf

Boude Figueredo, O (2013). Tecnologías emergentes en la educación: una experiencia de formación de docentes que fomenta el diseño de ambientes de aprendizaje. Educ. Soc, 34(123), 531-548.

Alcaldía Mayor de Bogotá. Secretaría de Educación (2012). Lineamientos de la Dirección de Ciencia, Tecnología y Medios Educativos. Disponible en: http://www. educacionbogota.edu.co/archivos/Temas $\% 20$ estrategicos/Documentos/Ciencia\%20y\%20 Tecnologia.pdf 
Aponte Correa, M.T. (2016). Consolidación de una comunidad de práctica para la incorporación de tecnologías de la información y comunicación en los colegios de ASPAEN. (Tesis de maestría).

Bennett, C. y Daniel, L. (1999). "Preparing novice teachers to use technology: Do they practice what we teach?" En: J. Price et al. (Eds.), Proceedings of Society for Information Technology and Teacher Education International Conference 1999 (pp. 11081111). Chesapeake, VA: AACE. 100

Canales, R. (2006). Identificación de factores que contribuyen al desarrollo de actividades de enseñanza y aprendizaje con apoyo de las TIC, que resulten eficaces y eficientes. Análisis de su presencia en tres centros docentes. Tesis Doctoral, Universidad Autónoma de Barcelona, Barcelona.

Castells, M. (1997). La era de la información: economía, sociedad y cultura, Vol. 1, Alianza, Madrid.

Correa, J.C. (2008). "Desafíos a la formación inicial del profesorado: buenas prácticas educativas en el contexto de la innovación con TIC". En: Revista Latinoamericana de Tecnología Educativa-RELATEC, 6(2), 109126.

Galvis, A. (2008). "La Piola y el desarrollo profesional docente con apoyo de Tecnologías de Información y Comunicación-TIC". En: Tecnología y Comunicación Educativas, 22(46), 59-86.

Hargreaves, A. (2003). Enseñar en la sociedad del conocimiento. Madrid: Octaedro.

Hernández, R., Fernández, C. y Baptista L. (2010). Metodología de la investigación ( $5^{a}$ Ed.). México: McGraw-Hill.

Instituto internacional de planeamiento de la educación (IIPE - UNESCO) (2006). Estado del arte y orientaciones estratégicas para la definición de políticas educativas en el sector. Buenos Aires: http://www.udelas.ac.pa/ biblioteca/librospdf/1_estadodelarte.pdf

Karsenti, T., y Gauthier, C. (2006). Les TIC bouleversent-elles réellement le travail des enseignants? Formation et profession, 12(3), 2-4.

Londoño, F., \& Castillo Peña, F. (2012). "PlanEsTIC: Un modelo para formulación de planes de incorporación de TIC en educación superior”. En: Memorias del XVII Congreso Internacional de Informática Educativa, TISE (pp. 132-138). Santiago de Chile: J. Sánchez.

Lucena, F.J.H.; Martín, F.D.F. \& Díaz, I.A. (2002). "Las actitudes de los docentes hacia la formación en tecnologías de la información y comunicación (TIC) aplicadas a la educación". En: Contextos educativos: Revista de educación, (5), 253-270.

Llorente Cejudo, M. del C. (2008). “Aspectos fundamentales de la formación del profesorado en TIC". En: Pixel-Bit: Revista de medios y educación, (31), 121-130.

Marcelo, C. (2001). "Aprender a enseñar para la Sociedad del Conocimiento". En: Revista Complutense de Educación, 2 (12), 531-593.

Ministerio de Educación Nacional (MEN). (2013). Competencias TIC para el desarrollo profesional docente. Disponible en: http:// www.colombiaaprende.edu.co/html/ micrositios/1752/articles-318264_recurso_ tic.pdf

(2013). "Competencias TIC para el desarrollo profesional docente". Sistema Nacional de Innovación Educativa con Uso de TIC. Colombia

Medina Rivilla, A.; Domínguez Garrido, M. y Ribeiro Gonçalves, F. (2011). "Formación del profesorado universitario en las competencias docentes". En: Revista Historia de la Educación Latinoamericana, 13(17), 119-138. 
Mejía, N. (2014). “Competencias en TIC de los docentesUniGuajira, Colombia”. En:Congreso Iberoamericano de Ciencia, Tecnología, Innovación y Educación. Disponible en: http:// www.oei.es/congreso2014/memoriactei/149. pdf

Ministerio de Educación Nacional (2010). Plan Decenal de Educación (2010-2016). Disponible en: http://www.plandecenal.edu. co/html/1726/articles-166057_TICS.pdf

Murillo Torrecilla, F.J. (2006). "Dirección escolar para el cambio: Del liderazgo transformacional al liderazgo distribuido". En: REICE: Revista Electrónica Iberoamericana sobre calidad, eficacia y cambio en educación, 4(4), 11-24.

Prieto, L. (2013). "Fortalecimiento de competencias digitales docentes a través de una modalidad E-Learning". En: Congreso de Investigación y pedagogía. Disponible en: http://tics.uptc.edu.co/eventos/index.php/ cong_inv_pedagogia/con_inv_pedag/paper/ view/199/196

Rivero, M.; García, C.I. y Ruíz, R. (2015). PlanEsTIC: Aprendizaje en comunidad para el fortalecimiento de la incorporación de TIC en educación superior en Colombia.

Robalino Campos, M. \& Koner, A. (2006). Modelos Innovadores en la Formación Inicial Docente: Estudio de casos de modelos innovadores en la formación docente en América Latina y Europa. Santiago de Chile: OREALC-UNESCO.

Rubio, M. J. y Escofet, A. (2013). "Estudio sobre los usos de las TIC y las posibilidades de empoderamiento en las mujeres". En: Revista Iberoamericana de Educación, 62(3), 1-13.

Silva,J.etal.(2006). “Estándares en tecnologías de la información y la comunicación para la formación inicial docente: situación actual y el caso chileno". En: Revista Iberoamericana de Educación (OEI), 2006, Vol. 38, No. 3.
Silva, C. y Loreto, M. (2004). "Empoderamiento: proceso, nivel y contexto". En: Revista Psykhe, 13(2), 29-39.

Swain, C. (2006). "Preservice teachers' selfassessment using technology: determining what is worthwhile and looking for changes in daily teaching and learning practices". En: Journal of Technology and Teacher Education, 14(1), 29-59.

Todd, Z.; Nerlich, B. \& McKeown, S. (2004). "Introduction". En: Z. Todd, B. Nerlich, S. McKeown \& D. Clarke (Eds.), Mixing methods in psychology (pp. 3-16). Hove, East Sussex, UK: Psychology Press.

Torres, A. (2009). "La educación para el empoderamiento y sus desafíos". En: Sapiens. Revista Universitaria de Investigación, 10 (1), 89-108. 